\title{
ARTICLE OPEN \\ Amorphous FeZr metal for multi-functional sensor in electronic skin
}

Minhyun Jung ${ }^{1}$, Eunha Lee ${ }^{2}$, Dongseuk Kim ${ }^{3}$, Kyungkwan Kim ${ }^{4}$, Changjin Yun ${ }^{4}$, Hyangsook Lee ${ }^{2}$, Heegoo Kim², Kungwon Rhie ${ }^{4}$ and Sanghun Jeon ${ }^{1}$

The composition of an amorphous metal, which is well known for having no slip plane or slip direction, can be tailored for enhanced strength and a lower Young's modulus under mechanical stress. Unlike crystalline metals, elastic amorphous metals can, in principle, be used as a flexible electrode in soft, wearable electronics. A metal with a lattice structure absorbs external energy, which causes structural deformations, while an amorphous metal does not absorb energy. Therefore, amorphous metals have excellent mechanical properties that can overcome the limitations of crystalline metals. However, voids inside such metals are easily formed according to Thornton's model, which results in the formation of mesoporous (10-50 nm) rods due to the spatial separation of metal atoms. We eliminated the porosity in amorphous metals by controlling the kinetic energy of sputtered metal atoms and ions. Optimized $\mathrm{Fe}_{33} \mathrm{Zr}_{67}$ has an amorphous structure with a strength of $2.88 \mathrm{GPa}$ and a Young's modulus of $76.6 \mathrm{GPa}$. With this amorphous metal, we fabricated a stretchable and multi-functional sensor for electronic skin that enables the detection of pressure, temperature and light and also serves as a heater. In this work, we demonstrate the feasibility of the amorphous metal $\mathrm{Fe}_{33} \mathrm{Zr}_{67}$ in soft electronics for use in various industrial applications.

npj Flexible Electronics (2019)3:8; https://doi.org/10.1038/s41528-019-0051-7

\section{INTRODUCTION}

An amorphous metal (e.g., metallic glass or liquid metal) is a metal that does not have a crystalline structure, unlike a typical crystalline metal (e.g., $\mathrm{Ti}, \mathrm{Au}$, or $\mathrm{Pt}$ ). All metals are processed into the desired shape at a temperature above the melting point. As the temperature is lowered, the atoms are arranged in a crystalline structure. Rapid cooling at a rate of more than $10^{6}$ degrees per second can result in a metal with an amorphous structure because the migration of atoms is limited prior to the cooled arrangement of the atoms. An amorphous metal exhibits physical properties that differ from those of a crystalline metal, and as a material, it has remarkable mechanical properties ${ }^{1-6}$ and processability. ' Due to the advantages of the amorphous structure, amorphous metals have become an option for soft electronics (stretchable and wearable electronics). Since the discovery of amorphous metals, ${ }^{8}$ more than 30 atomic elements have been reported as constituents of amorphous metal-forming alloys, including most transition metals and alkali metals. ${ }^{9-12}$ Research on amorphous metals has generally progressed from a narrow range of eutectic systems with a trial-and-error approach. Amorphous metals can also be alloys made from metals with different atomic radii. Alloys of Fe compositions in particular have been studied in many fields due to their high conductivity and excellent bonding with other materials. ${ }^{13}$

Electronic skin (e-skin) is an assembly of sensors that sense various physical and chemical stimuli. These sensors are similar to the sensory receptors in human skin that deliver various tactile and thermal signals to the brain. Although research on e-skin that mimics the various structures and senses found in nature, including those in the human body, is ongoing, developing eskin with high durability and various detection devices remains a challenge. Flexible sensors will inevitably be required for bodyattachable devices, and research has been carried out such as metal wave structures, ${ }^{14}$ conductive polymers, ${ }^{15-18}$ embedded nano particle, ${ }^{19}$ and biocompatible material with pyramidal structure $^{20}$ on a flexible platform. Highly reliable and flexible eskin with various detection modes may be used in the healthcare and humanoid robot areas. ${ }^{21-23}$ In order to realize such devices, a high-performance, high-reliability, body-attachable health monitoring system is required. However, most previous reports did not describe the detection of various stimuli (such as pressure, temperature, or optical), and the problems associated with the reliability of organic materials and several problems pertaining to uniformity remain to be solved.

Here, we propose a high-performance, multifunctional (pressure, temperature, optical sensor, and heater) device made of an amorphous metal, which is suitable for wearable electronics that must maintain integrity even under external types of force such as stretching and bending. We deposited the $\mathrm{Fe}_{33} \mathrm{Zr}_{67}$ (atomic percent; at\%) amorphous alloy, which has excellent mechanical properties and retains certain electrical properties under physical stimulation such as bending or stretching on a flexible substrate. We secured the feasibility of e-skin using amorphous metal-based resistance-changing pressure sensors, temperature sensors, and photosensors based on In-Zn-O thin film transistors to check the health status of a user. We also fabricated a micro-heater to inject

\footnotetext{
${ }^{1}$ Department of Electrical Engineering, Korea Advanced Institute of Science and Technology, Daehak-Ro 291, Yuseong-Gu, Daejeon, Republic of Korea; ${ }^{2}$ Analytical Engineering Group, Platform Technology Laboratory, Samsung Advanced Institue of Technology, Suwon 16678, Republic of Korea; ${ }^{3}$ Spin Convergence Research Team, Korea Research Institute of Standards and Science, Daejeon 34113, Republic of Korea and ${ }^{4}$ Department of Applied Physics, Korea University, Sejong 30019, Republic of Korea Correspondence: Kungwon Rhie (krhie@korea.ac.kr) or Sanghun Jeon (jeonsh@kaist.ac.kr)

These authors contributed equally: Minhyun Jung, Eunha Lee, Dongseuk Kim
}

Received: 29 May 2018 Accepted: 18 March 2019

Published online: 18 April 2019 
appropriate medicine. This e-skin, which integrates a multipurpose sensor capable of excellent performance, can be used in a wide range of applications, such as human-machine interactive interfaces, healthcare-monitoring systems, and even medical diagnostic devices.

\section{RESULTS AND DISCUSSION}

With various experiments, we demonstrated that amorphous metals are suitable for use in flexible electronics. Metals with a general lattice structure have long-range-ordered grain boundaries that allow electrons to move freely, and they exhibit high levels of electrical and thermal conductivity. In addition, the crystal structure of these metals has a slip plane and a slip direction. Owing to these physical properties, structural deformations occur along the slip plane when a certain level of external force is applied. However, amorphous metals consist mainly of alloys composed of two or more materials, with atoms of different atomic radii. Further, the electro-mechanical properties of amorphous metals differ from those of crystal metals due to their indiscriminate arrangements of atoms.

As shown in Fig. 1a, the discontinuity of a crystalline metal begins at the slip plane of the lattice with the application of external force. However, because an amorphous metal does not have a lattice structure, atomic deformation occurs but the metal does not break easily (see Fig. 1b). To evaluate the outstanding mechanical properties of the amorphous metal investigated here, we compared FeZr thin films with a general crystalline metal. For the comparison, the thickness of all metal thin films used in the experiment was fixed at $50 \mathrm{~nm}$. Figure 1c, d depict surface images of titanium and amorphous metal FeZr thin films after bending. Because the Ti thin film has a lattice structure, large cracks form after external stress is applied. These cracks were confirmed by optical microscopy (SEM images are also presented in Supplementary Information S1). Titanium thin film is difficult to use as a metal electrode after bending owing to its high resistivity stemming from such large cracks. However, the FeZr thin film showed no significant changes in the metal resistance, and no critical cracks were observed, even after bending. In Fig. 1e, f, the mechanical properties of the amorphous metal FeZr were found to be the best among various metals in terms of the sheet resistance in the bending and elongation tests (see the Supplementary Information Video S1).

According to the Thornton model, we can classify the film microstructure with a zone model upon sputtering with different argon pressures, melting temperatures of the target, and substrate temperatures. The growth of the FeZr thin film fits the Thornton model. The film deposited at a low pressure level has a dense structure with a smooth surface morphology while the film deposited at a high pressure level has a columnar structure with a rough surface and voids between the columns. ${ }^{24}$ However, this model does not explain the physical process of each type of growth structure, and it stems wholly from experimental results. ${ }^{25}$ For a clearer understanding of thin film growth, we considered three basic modes of atomic growth: the Volmer-Weber (island growth), Stranski-Krastanov (layer with island growth), and Frankvan der Merwe (layer growth) modes.

At high pressures, the mean-free paths of Fe and $\mathrm{Zr}$ atoms are short as their potential to interact with argon atoms increases. In addition, this condition decreases the mobility of atoms at the surface of the growing film, leading to a void structure due to a shadowing effect. ${ }^{26}$ Due to the low kinetic energy, atoms start to interact with each other and form atomic clusters, leading to the formation of a columned structure with randomly located voids according to the Volmer-Weber mode (see Fig. 2a). In contrast, at intermediate pressures, dense FeZr is formed on the surface of the substrate. However, it gradually grows with a columned structure in the Stranski-Krastanov mode (see Fig. 2b). Moreover, a compact amorphous structure is formed by a long mean-free path which arises due to the high kinetic energy of the atoms deposited at a low pressure. This condition decreases the amount of interaction between the deposited atoms with argon, leading to the formation of a smooth surface of thin film. It also allows sputtered atoms to be adsorbed to the surface of the film and prevents them from diffusing to form an equilibrium lattice. Deposition into an amorphous structure with an alloy metal is easy because each element effectively suppresses the atomic mobility of the other atom. In such a case, the amorphous film becomes a uniformly arranged film without voids or grain boundaries in what is known as the Frank-van der Merwe mode (see Fig. 2c). Thus, the growth structure of the film depends on the deposition pressure (see Fig. 2d-f).

In our study, to investigate the amorphous metal film structure considering the kinetic energy of sputtered atoms, we deposited $50 \mathrm{~nm}$ FeZr films at different working pressures of 3, 7, and 11 mTorr. Here, we analyzed the structure of the FeZr thin film under the different pressures and adjusted the void structure inside the thin film in each case. We also present cross-sectional views of the three samples. High-angle annular dark-field scanning transmission electron microscopy (HAADF-STEM) images revealed that the FeZr thin film deposited at a high pressure (Fig. $2 \mathrm{~g}$ ) has a void structure between the columns. The film deposited at intermediate pressures (Fig. 2h) has a mixture of compact and void structures. In contrast, the thin film deposited at a low pressure (Fig. 2i) has a compact amorphous structure.

These results indicate that the film structure changes gradually from a column with voids to a compact amorphous structure as the kinetic energy of the deposited atoms increases. Metal thin films having a void structure have weak mechanical properties because fractures can easily form in the columns inside the thin film when external force is applied. However, the FeZr thin film with a perfect amorphous structure shows excellent mechanical characteristics under different types of stress, such as bending and stretching. The electrical hysteresis of the FeZr thin film was less than $10 \%$ when the film was stretched by $20 \%$ (see Supplementary Information S2 and S3).

In an additional material analysis of FeZr, we characterized the structure of the amorphous metal by means of an energydispersive X-ray spectroscopy analysis with different deposition pressures using HAADF-STEM (see Fig. 3). We were able to control the size and thickness of the pores in the FeZr thin film while reserving the amorphous structure. As shown in Fig. 3a, b, FeZr, which is an amorphous metal, forms a complete amorphous structure because it is deposited on a substrate with high energy during sputtering when the deposition pressure is low. However, we confirmed that at higher deposition pressures, more porous voids are formed between the surface and the interface with the substrate (see Fig. 3c, d). We characterized transmission electron microscope (TEM) images under two deposition conditions to confirm whether the amorphous metal develops a crystalline structure or retains an amorphous structure as the deposition pressure is increased. Fast Fourier transform of the TEM images confirmed that the FeZr thin film deposited at a high kinetic energy level has a compact amorphous structure, whereas the opposite case has a partially crystalline structure. By using these properties of the compact-amorphous-structured FeZr, it is possible to form a structure having excellent mechanical characteristics under external different types of physical stress, such as stretching and bending (see Supplementary Information Figs. S4 and S5).

Subsequently, we measured the performance capabilities of our amorphous metal-based multi-mode sensor. First, for a pressure sensor, we used micro-pyramid-structured polydimethylsiloxane (PDMS). An amorphous FeZr metal layer was deposited onto the PDMS microstructure by a sputtering process. Then, FeZr was deposited onto the back of polyethylene terephthalate (PET) for 
(a)

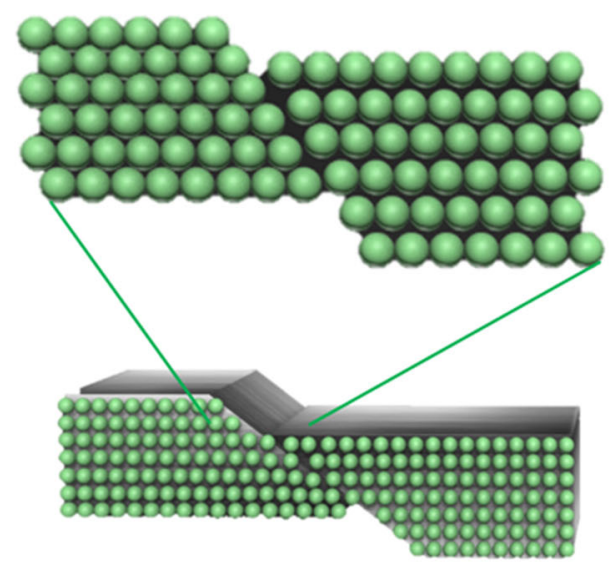

(c)

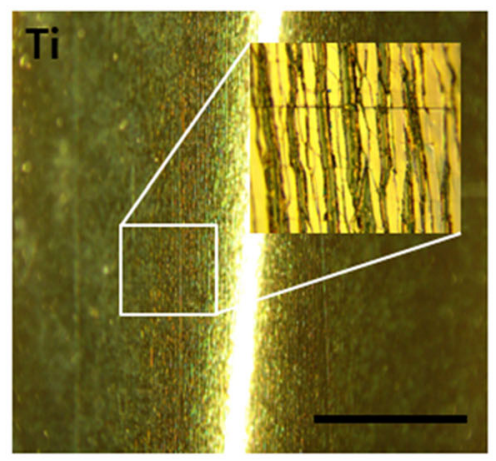

(e)

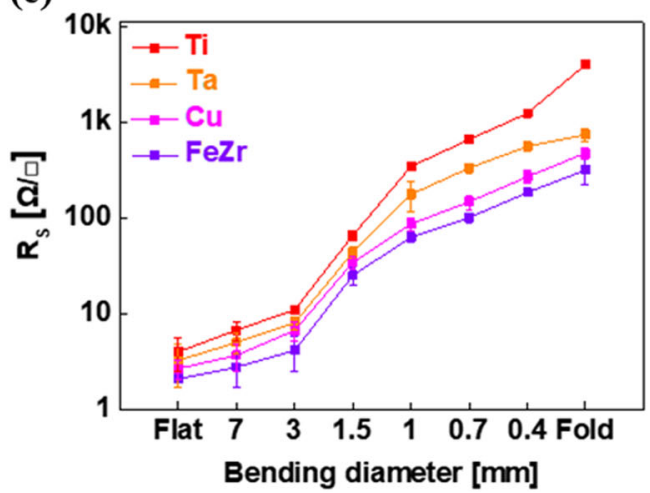

(b)

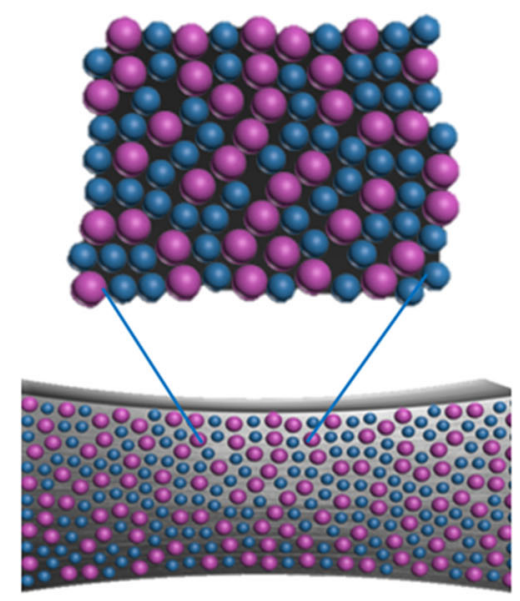

(d)

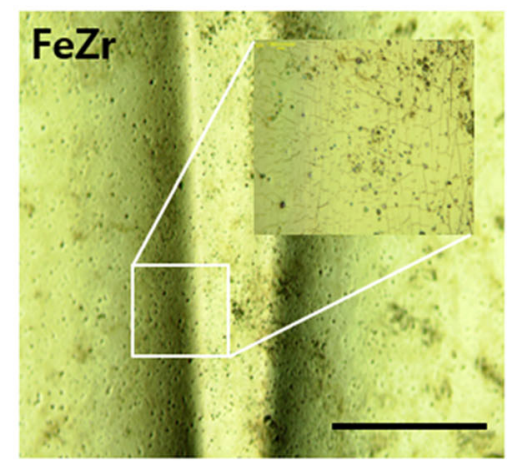

(f)

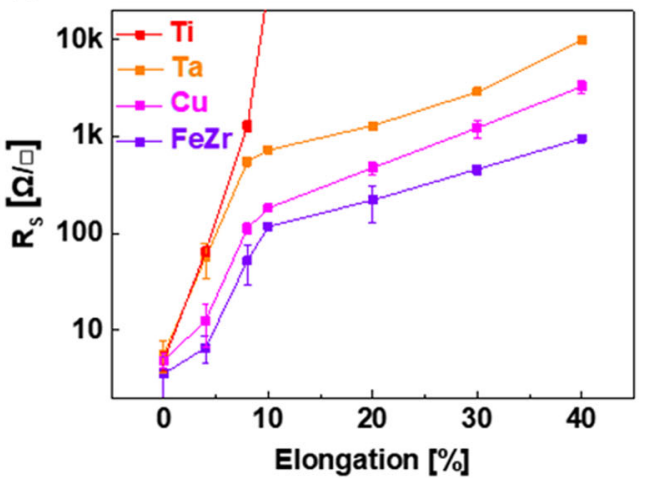

Fig. 1 Comparison of amorphous metal and crystalline metal. Structural deformation diagram according to external force of a crystalline metal and $\mathbf{b}$ amorphous metal. Optical images of $\mathbf{c ~ T i}$ and $\mathbf{d}$ FeZr thin film under bending situation. Inset shows magnified view. Scale bar is $1 \mathrm{~mm}$. Sheet resistance of various metal thin films with e bending diameter and $\mathbf{f}$ elongation

use as a counter electrode. Given that a PDMS microstructure is flexible, the pyramidal structure will be deformed under mechanical stimuli. With the change in the contact area between the upper electrode and the lower electrode, we can measure the current change out of the pressure sensor. The current-change characteristics of the pressure sensor can be expressed as Eq. 1.

$\Delta I=\frac{1}{\rho} \Delta \frac{V_{\mathrm{op}} A_{\mathrm{C}}}{L_{\mathrm{CA}}}$,

From the perspective of physical geometry, the length of the contact area between the top electrode and the pyramid structure, $\left(L_{C A}\right)$ and the contact area $\left(A_{C}\right)$ of the deformed pyramid change due to external force. Consequently, when the operating voltage $\left(V_{\mathrm{op}}\right)$ and the resistivity of the deposited metal $(\rho)$ are constant, the resistance of the contact area will change with external force, and a current change due to this geometric deformation will be detected. A wide range pressure-sensing capabilities and rapid response speeds are essential to realize a type of electronic skin that mimics human tactile perception. Figure $4 a$ (the inset in the figure shows a schematic view of the pressure sensor) shows the performance of our pressure sensor in a range of $100 \mathrm{~Pa}-5 \mathrm{kPa}$. The linear current-voltage curves show ohmic behavior with different loading pressures. With an increase in the loading pressure, the current level increased as well. A fast response time $(\sim 25 \mathrm{~ms})$ was also noted, as shown in Supplementary Information Fig. S6. Figure $4 \mathrm{~b}$ also indicates that it is possible 
(a)

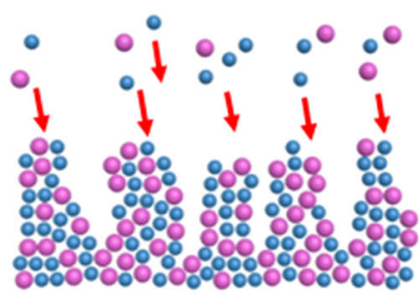

(d)

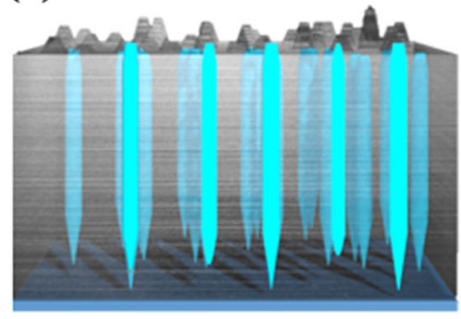

(g)

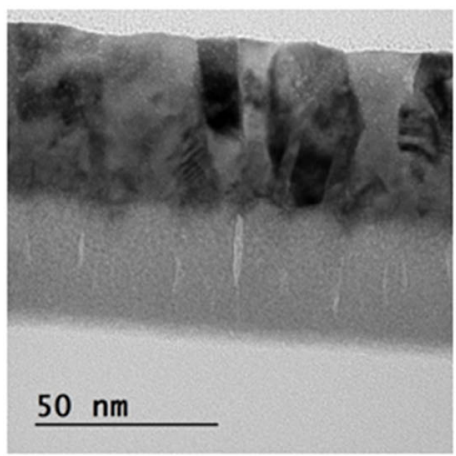

(b)

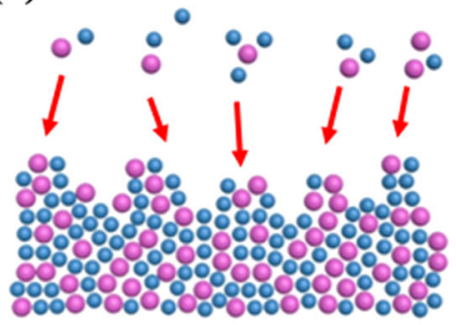

(e)

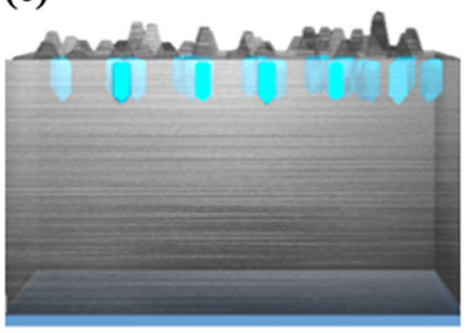

(h)

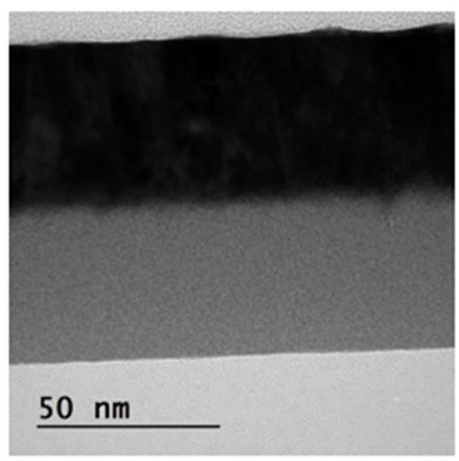

(c)

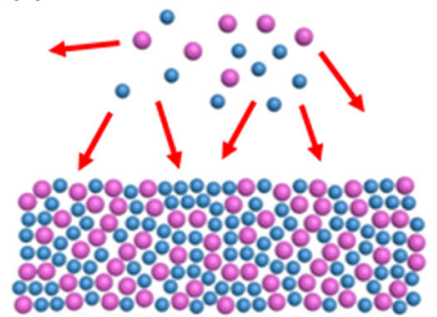

(f)

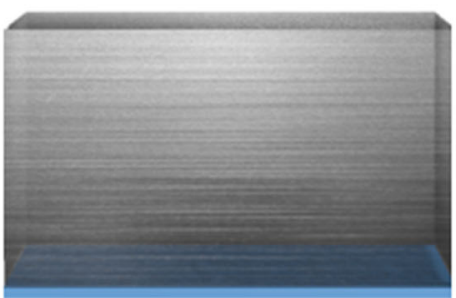

(i)

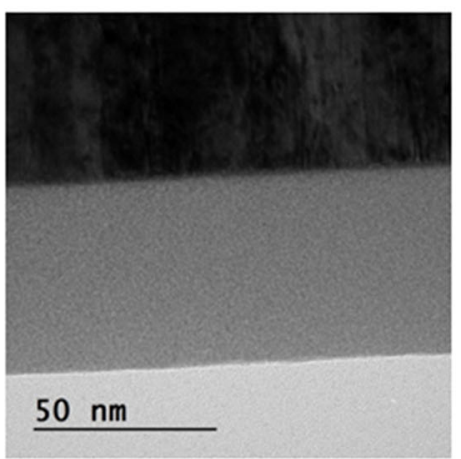

Fig. 2 Growth mode and analysis of amorphous metal thin film. Schematic diagram of amorphous metal layer with different growth modes. a Volmer-Weber mode which has column structure, b Stranski-Krastanov mode with partial voids, and c Frank-van der Merwe mode with compact amorphous structure. Schematic of atomic growth with $\mathbf{d}$ low, e intermediate, and $\mathbf{f}$ high kinetic energy. TEM images of FeZr thin film with different sputtering pressure w/ Pt capping layer. g $11 \mathrm{mTorr}$, h $7 \mathrm{mTorr}$, and $\mathbf{i} 3 \mathrm{mTorr}$, respectively

to measure the heart rate with a skin-attached amorphous metalbased pressure sensor. This figure depicts the measurement of the peak values of a typical pulse (see Supplementary Information Video S2). We can check the health condition of the user using the blood flow amplitude $P_{1}$ and reflected wave $\left(P_{2}\right.$ : wrist, $P_{3}$ : lower body) due to the contraction of the heart muscle. For example, the radial artery elevation index (Alr $=P_{2} / P_{1}$ ), radial diastolic augmentation $\left(\mathrm{DAI}=P_{3} / P_{1}\right)$, and the travel time of waves reflected from the hand $\left(T_{\mathrm{R}}\right)$ are commonly used for a quantitative evaluation of atherosclerosis. $^{27}$ In previous reported stretchable electronics, metallic interconnection with various structures were adopted. ${ }^{28}$ However, in this case, it is hard to avoid increase in resistance due to the increase in length of the electrode. In our approach, we used fully deposited FeZr as a conductive layer and can be operated at extremely low voltages $(\sim 1 \mathrm{mV})$. High-performance and low-power-consumption characteristics are suitable for wearable devices with limited power capacities. Figure 4c, d show the high durability $\left(\sim 10^{5}\right)$ of the FeZr pressure sensor in comparison with devices manufactured using crystalline metals ( $\mathrm{Ta}$, Ti). The response/relaxation curves of the pressure sensor were evaluated for 100,000 cycles at $1 \mathrm{kPa}$. During the repeated cycles, there was a degradation of current value less than $15 \%$ of the initial state. These results demonstrate the outstanding performance of the proposed sensor. To evaluate the performance of our pressure sensor as an acoustic sensor, we measured the response of the pressure sensor to various sounds, as shown in Fig. $4 \mathrm{e}$, and the response to music as well, as demonstrated in Fig. 4f. These results indicate that our sensors can be applied to a variety of applications. (Measurement schemes are shown in Supplementary Information Fig. S7)

Furthermore, we fabricated a temperature sensor with an amorphous metal thin film layer next to the pressure sensor on the same plane. Because we can make these sensors tiny and given that these components are integrated, we can introduce the concept of multimodal sensor capable of detecting pressures as well as temperatures at the same time (see Fig. 5a). The crystalline metal layer exhibits a positive temperature coefficient on resistance (TCR), indicating that the resistance increases with the temperature. However, because an amorphous metal does not have a lattice structure, the initial resistance of an amorphous metal is higher than that of a crystalline metal layer, but its resistance decreases with an increase in the temperature, indicating that FeZr has a negative TCR. ${ }^{29}$ This phenomenon is repeatable unless the amorphous structure is ordered in a lattice structure at temperatures above $500^{\circ} \mathrm{C}$. Thus, we have experimentally demonstrated that an amorphous metal can function as a temperature sensor (see Fig. 5b). This result shows that the FeZr temperature sensor is most sensitive with an absolute TCR value of 
(a)

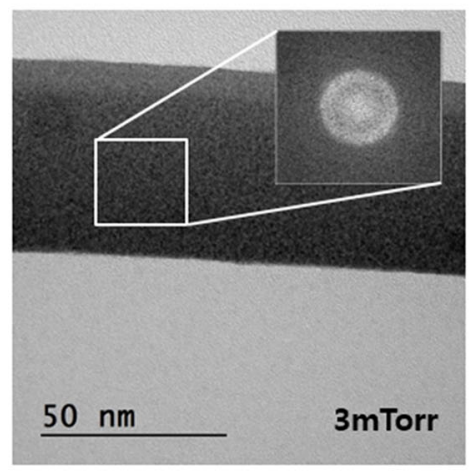

(c)

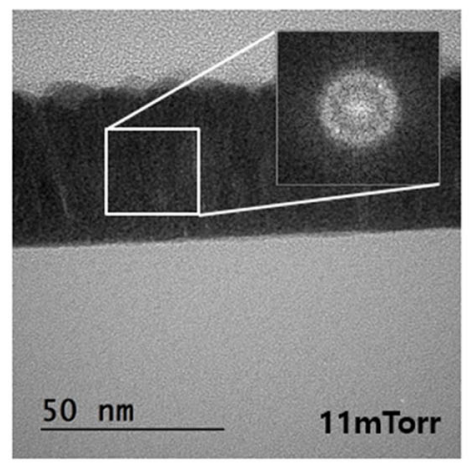

(b)
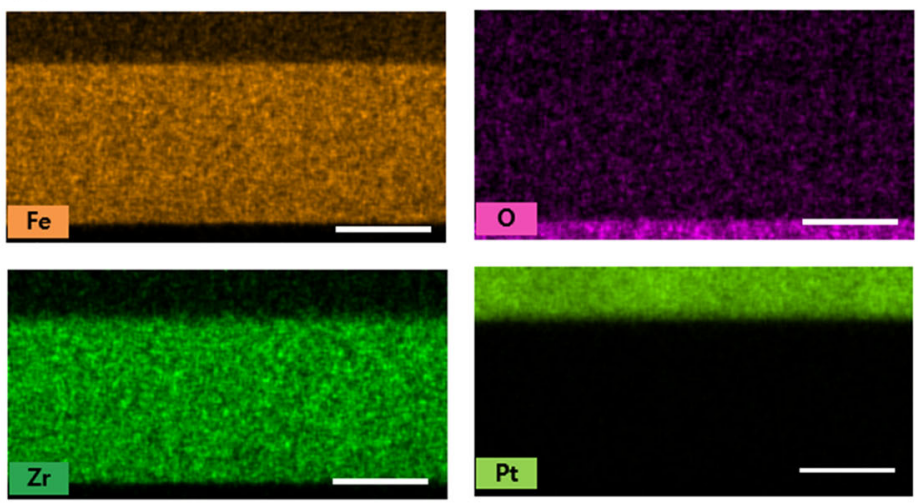

(d)
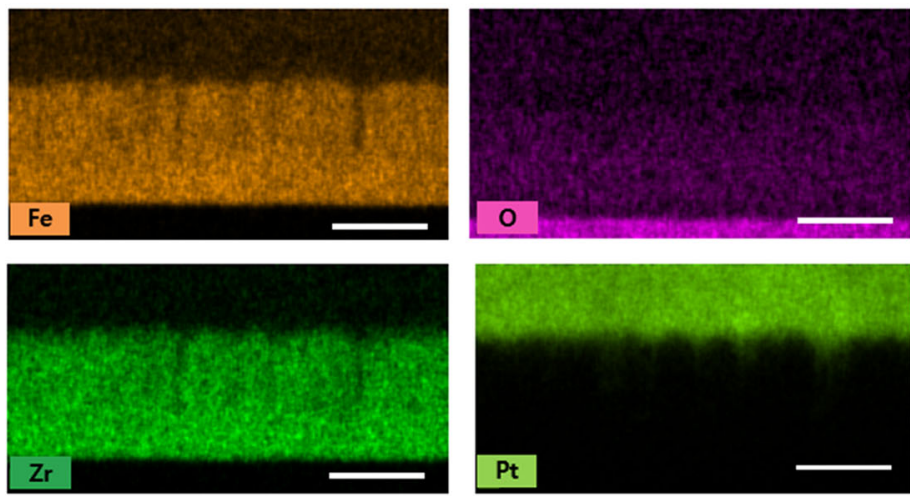

Fig. 3 Analysis of structural properties of amorphous metal. a The transmission electron microscope (TEM) image of FeZr deposited at $3 m$ Torr. Inset shows the fast-Fourier-transform (FFT) data. b The energy dispersive X-ray spectroscopy (EDS) data of FeZr deposited at 3mTorr. Scale bar is $30 \mathrm{~nm}$. c TEM image (inset shows the FFT data) and d EDS data of FeZr deposited at $11 \mathrm{mTorr}$. Scale bar is $30 \mathrm{~nm}$

$5.30405 \Omega /{ }^{\circ} \mathrm{C}$ in comparison with gold $\left(1.1854 \Omega /{ }^{\circ} \mathrm{C}\right)$ and platinum $\left(0.81561 \Omega /{ }^{\circ} \mathrm{C}\right)$. Moreover, the experimental results indicate a linear shape curve for a dynamic range of $25-150^{\circ} \mathrm{C}$. In addition to measuring the temperature-sensing performance of $\mathrm{FeZr}$, we also produced a heater that can inject drugs into humans through the skin in the form of a micro-needle or a capsule that reacts at a certain temperature. To demonstrate the performance of the amorphous metal as a heater, FeZr thin film was deposited onto PET and voltage of $10 \mathrm{~V}$ was applied. The temperature was then measured over time, as shown in Fig. $5 \mathrm{c}$. We found that the temperature was raised and then became saturated at $80^{\circ} \mathrm{C}$. This result indicates that the thin film can sufficiently operate as a heater (see Supplementary Information Fig. S8).

We also utilized the amorphous FeZr thin film as metal electrodes for a photo-thin film transistor (TFT). The photo-TFT based on In-Zn-O (IZO) is known to be a device with excellent photoreactivity. ${ }^{30,31}$ The high optical absorption of the IZO layer can be attributed to carrier generation, causing a drastic increase in the photocurrent of the IZO TFT at $400-550 \mathrm{~nm}(2.2-3.1 \mathrm{eV})$. Unfortunately, however, research on stretchable gate insulate and the channel materials for transistor is still in progress. In this study, we focused on the feasibility of amorphous metal as an electrode of flexible electronic devices. Therefore, we fabricated IZO TFT individually on rigid silicon wafer and electrically connected amorphous FeZr $(50 \mathrm{~nm})$ on PDMS was performed as a stretchable electrode. As a result, we were able to measure the characteristics of an IZO photo sensor with FeZr electrodes which yielded a saturation mobility of $3.12 \mathrm{~cm}^{2} \mathrm{eV}^{-1} \mathrm{~s}^{-1}$, a threshold voltage level of $4 \mathrm{~V}$, and a subthreshold swing of $810 \mathrm{mV} /$ decade in a dark state (drain voltage $10 \mathrm{~V}$ ). Figure $5 \mathrm{~d}$ shows the transfer characteristics of the photosensor with a channel width and length of $100 \mu \mathrm{m}$ and $50 \mu \mathrm{m}$, respectively. The dependence of the photo-TFTs was measured under light wavelengths of 550, 500, 450, and $400 \mathrm{~nm}$ and at a power of $200 \mu \mathrm{Wcm}{ }^{-2}$. The experimental result shows that IZO TFTs with FeZr electrodes are sensitive to specific wavelengths. In addition, our TFT retained a certain level of electrical characteristics with an on current of $1.4 \mu \mathrm{A}$ when the FeZr metal electrodes were stretched $15 \%$ (see Fig. 5e). Most previously reported stretchable devices have been carried out with structural aspects such as zig-zag, horseshoe and wrinkling rather than elongation of the material itself. ${ }^{32,33}$ This result clearly demonstrates the feasibility of FeZr for use as a core material in stretchable electronics created using conventional semiconductor fabrication processes.

As mentioned above, we can diagnose health condition of user through the data acquired by our device and detect external physical stimuli such as pressure, temperature, and light. Further, an implemented heater on a flexible platform can also inject a drug through the skin to the user. In addition to evaluating the comprehensive performance of a single device, we also evaluated an array of multimode sensors and utilized them as e-skin (see Supplementary Information Fig. S9). The real-time mapping results indicate that pressure and temperature in a $5 \times 5$ array system can be successfully monitored. Despite the fact that the pressure and temperature sensors use the same resistance change principle, we applied a vertically stacked structure, and each signal was received separately. As a result, distributions of both pressures and 
(a)

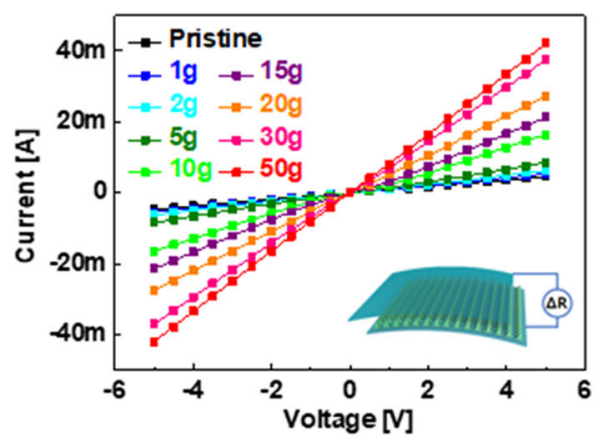

(c)

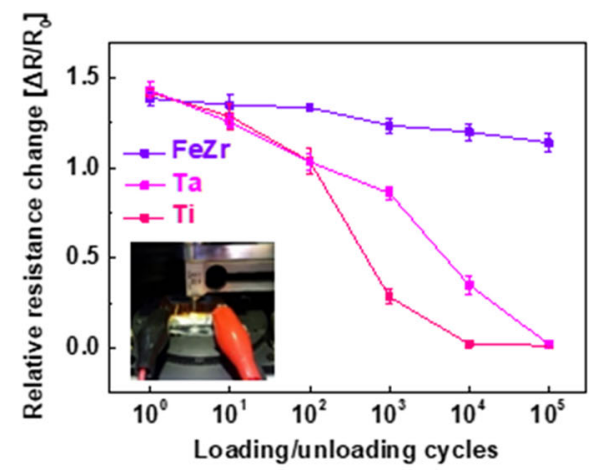

(e)

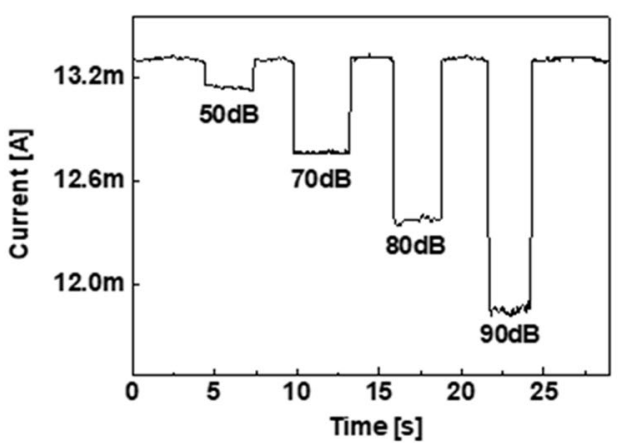

(b)

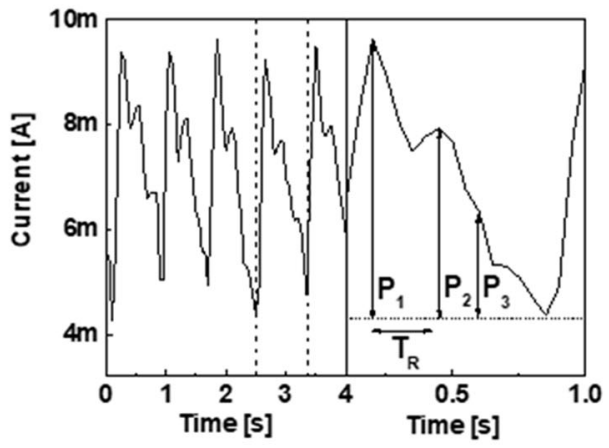

(d)

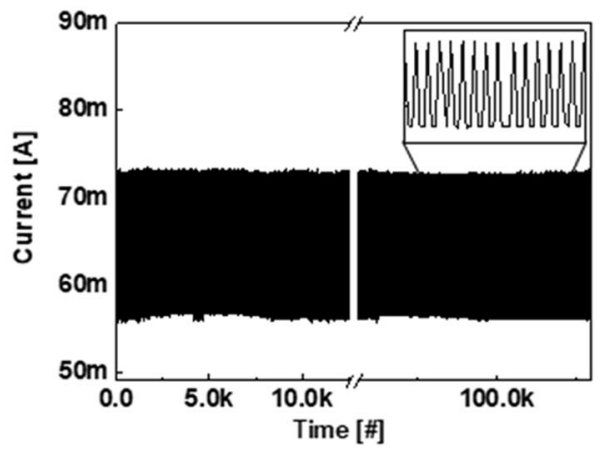

(f)

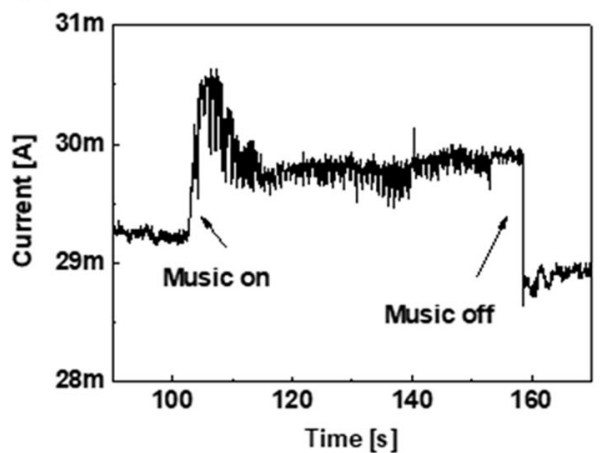

Fig. 4 Pressure sensing performance of amorphous metal-based pressure sensor. a The pressure sensing performance with different applied force (Unit area: $\left.5 \mathrm{~mm}^{2}\right)$. b The heart beat rate measurement data using pressure sensor $\left(P_{1}\right.$ : blood flow amplitude and reflected wave from $P_{2}$ : wrist, $P_{3}$ : lower body). c Relative resistance changes of pressure sensor with various metals and $\mathbf{d}$ repeatability characteristic of FeZr pressure sensor show the reliability of pressure sensing performance. The electrical characteristics of pressure sensor as sound detection sensor under e various magnitude of sounds and $\mathbf{f}$ music

temperatures applied from the same area can be detected without interference.

The low Young's modulus of amorphous bulk metals results in negligible structural deformation under mechanical stress. However, void in amorphous metal films can form easily according to Thornton's model. By adjusting the incident ion energy of a sputtered metal, we eliminated the porous rods in amorphous metals. In this work, we analyzed the physical and mechanical properties of an amorphous metal thin film while considering several sputtering process parameters. FeZr metal film deposited at a low pressure ( 3 mTorr) presents a perfectly amorphous structure without pores, thereby showing outstanding physical and electrical properties even under mechanical forms of stress such as stretching and bending. We employed an optimized amorphous FeZr electrode as a soft and wearable tactile sensor, a heater, and as a healthcare patch. In addition, this material was utilized as the core electrode of a photo sensor and switched transistor devices. Our amorphous FeZr-integrated sensor formed on a flexible platform presents a high range of pressure sensitivity $(100 \mathrm{~Pa}-5 \mathrm{kPa})$, temperature sensitivity $\left(\sim 150^{\circ} \mathrm{C}\right)$ photo responsivity $(400-550 \mathrm{~nm})$ levels and can function as a high-performance heater $\left(\sim 80^{\circ} \mathrm{C}\right)$ that can be used for comprehensive diagnostics and drug infusion applications. The results presented here indicate that an amorphous metal is well suited for use in soft, wearable, and multi-functional electronics. We expect that amorphous metals and amorphous metal-integrated devices will be innovative elements in soft electronics such as healthcare patches and e-skin. 


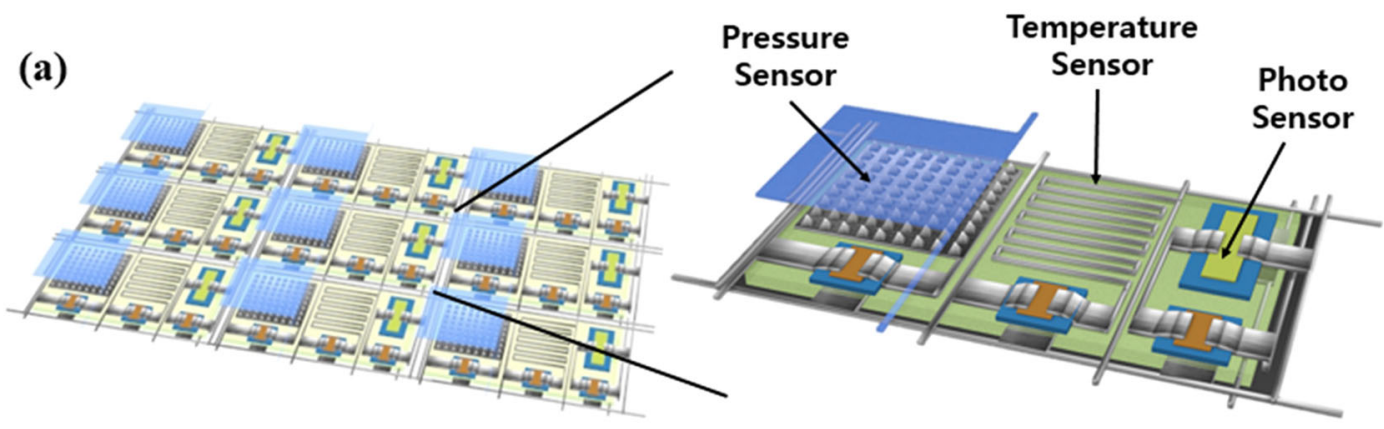

(b)

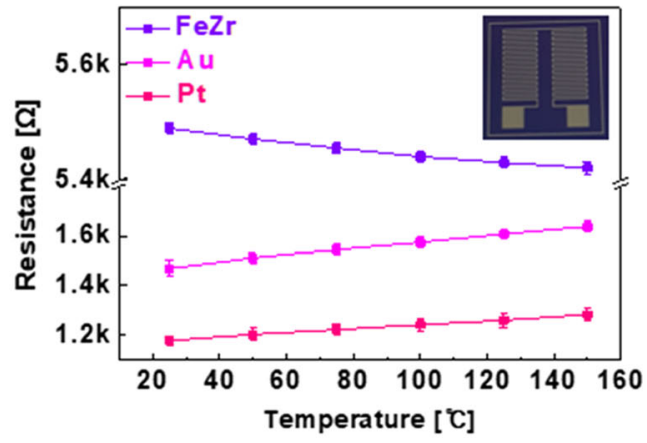

(d)

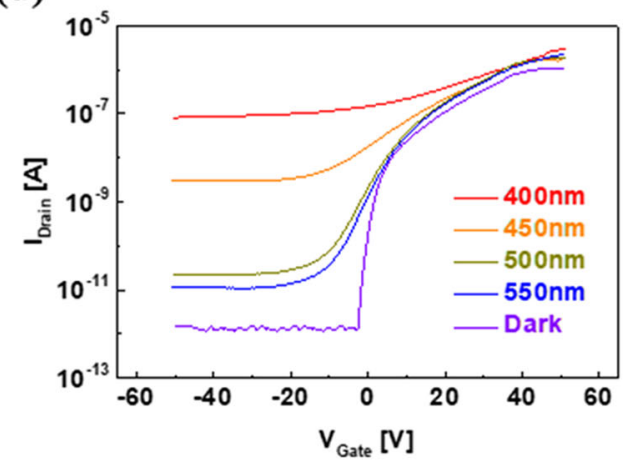

(c)

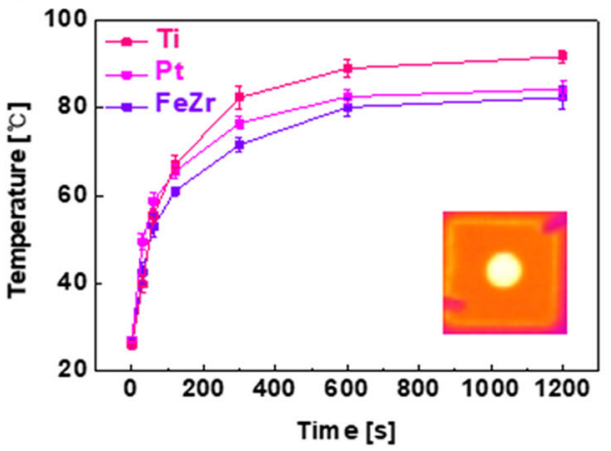

(e)

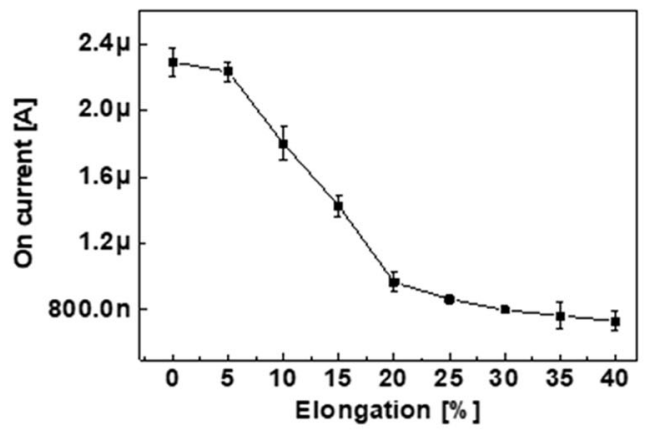

Fig. 5 Characteristics of amorphous metal based multimodal sensor. a Conceptual schematic of multi-functional sensor array. $\mathbf{b}$ Temperature sensing and $\mathbf{c}$ heater performance of various metal thin films. Electrical characteristics of In-Zn-O thin film transistor with amorphous metal electrodes under $\mathbf{d}$ illumination with light wavelengths and e elongation stress

\section{METHODS}

Device fabrication

To fabricate the mold with the pyramid structures, various sizes of squares were patterned on a silicon substrate with photolithography and etched for $1 \mathrm{~h}$ at $90^{\circ} \mathrm{C}$ in $\mathrm{KOH}$ aqueous solution (44 wt\%). After making the silicon mold, PDMS was poured, and it was left in a vacuum jar for $2 \mathrm{~h}$ to eliminate air bubbles. Then, PDMS was cured by thermal annealing process for $1 \mathrm{~h}$ at $80^{\circ} \mathrm{C}$. The PDMS was peeled off the silicon mold and cleaning was carried out using IPA. The amorphous metal FeZr $(50 \mathrm{~nm})$ as the conductive layer for the multifunction sensor was deposited on the PDMS using a DC magnetron sputtering system (RF power: $50 \mathrm{~W} /$ 3 mTorr) under a patterned shadow mask. The temperature sensor was designed to have a comb-like pattern for optimal effect and the FeZr was deposited next to the pyramid structure. The photo sensor was performed with IZO phototransistor. P-type silicon wafer $\langle 100\rangle$ with thermal oxide layer $(300 \mathrm{~nm})$ was prepared as a substrate. Then, the IZO $(40 \mathrm{~nm})$ layer was deposited using an RF sputtering system (DC voltage: $300 \mathrm{~V} / \mathrm{RF}$ power: $100 \mathrm{~W} / 5 \mathrm{mTorr}$ ). After photo lithography process for pattering the channel layer, the metal electrodes and line for all transistors consisted of FeZr (50 nm).

\section{Characterization}

Surface images for the detailed material analysis of various metal thin films were observed by field-emission scanning electron microscopy (Tescan MIRA3). The electrical characteristics of the amorphous metal thin film were evaluated using a customized bending and stretching machine and a 4-point probe system with LabVIEW. The performances of the multifunctional sensors were measured using a universal measurement probe system (Teraleader UMP-1000) and a source meter (Keithley 2401). In order to evaluate and characterize the performance of the temperature and photo sensor, performance measurements were carried out with a semiconductor parameter analyzer (Agilent $4156 \mathrm{C}$ ) and a custom-made temperature control system. The thermal images for evaluating the temperature dependence of the heater and temperature sensor were measured using an infrared camera (FLIR C2). The electrical characteristics of the photo sensor with different wavelength were evaluated using a wavelength spectrometer (Uni nanotech).

\section{DATA AVAILABILITY}

All data generated or analyzed during this study are included in this published article (and its supplementary information files). 


\section{ACKNOWLEDGEMENTS}

This work was supported by the Future Growth Engine Program (10079974, Development of core technologies on materials, devices, and processes for TFT backplane and light emitting front plane with enhanced stretchability above $20 \%$, with application to stretchable display) funded by the Ministry of Trade, Industry \& Energy (MOTIE, Korea). Also, this work was supported by the Technology Innovation Program (or Industrial Strategic Technology Development Program (10049163) funded By the Ministry of Trade, Industry \& Energy (MOTIE, Korea). Also, this work was supported by the BK21 plus program through the National Research Foundation (NRF) funded by the Ministry of Education of Korea.

\section{AUTHOR CONTRIBUTIONS}

M.J. and E.L. equally contributed to the manuscript. M.J. and C.Y. carried out experimental work with the contribution from D.K. and analyzed the data with contributions from E.L., H.L., H.G.K.; M.J., C.Y. and K.K. contributed to sample preparation. S.J. and K.L. designed and directed the project; The manuscript was written by M.J. and S.J. with contributions from E.L., K.K., C.Y., K.L., H.L., H.K. and D.K.; All authors discussed the results and contributed to the final manuscript.

\section{ADDITIONAL INFORMATION}

Supplementary information accompanies the paper on the npj Flexible Electronics website (https://doi.org/10.1038/s41528-019-0051-7).

Competing interests: The authors declare no competing interests.

Publisher's note: Springer Nature remains neutral with regard to jurisdictional claims in published maps and institutional affiliations.

\section{REFERENCES}

1. Ding, S. et al. Combinatorial development of bulk metallic glasses. Nat. Mater. 13, 494-500 (2014).

2. Inoue, A., Shen, B., Koshiba, H., Kato, H. \& Yavari, A. R. Cobalt-based bulk glassy alloy with ultrahigh strength and soft magnetic properties. Nat. Mater. 2, 661-663 (2003).

3. Pauly, S., Gorantla, S., Wang, G., Kühn, U. \& Eckert, J. Transformation-mediated ductility in CuZr-based bulk metallic glasses. Nat. Mater. 9, 473-477 (2010).

4. Demetriou, M. D. et al. A damage-tolerant glass. Nat. Mater. 10, 123-128 (2011).

5. Liu, Y. H. et al. Super plastic bulk metallic glasses at room temperature. Science 315, 1385-1388 (2007).

6. Si, J., Mei, J., Wang, R., Chen, X. \& Hui, X. Fe-B-Si-Zr bulk metallic glasses with ultrahigh compressive strength and excellent soft magnetic properties. Mater. Lett. 181, 282-284 (2016).

7. Kumar, G., Tang, H. X. \& Schroers, J. Nanomoulding with amorphous metals. Nature 457, 868-872 (2009).

8. Klement, W., Willens, R. \& Duwez, P. Non-crystalline structure in solidified gold-silicon alloys. Nature 187, 869-870 (1960).

9. Johnson, W. L. Bulk glass-forming metallic alloys: science and technology. MRS Bull. 24, 42-56 (2013).

10. Wang, W. H. Roles of minor additions in formation and properties of bulk metallic glasses. Progress. Mater. Sci. 52, 540-596 (2007).

11. Wang, W. H., Dong, C. \& Shek, C. H. Bulk metallic glasses. Mater. Sci. Eng. 44, 45-89 (2004).

12. Inoue, A. Stabilization of metallic supercooled liquid and bulk amorphous alloys. Acta Mater. 48, 279-306 (2000).

13. Sumiyama, K. \& Nakamura, Y. Magnetic properties of metastable crystalline and amorphous Fe alloys produced by RF sputtering. J. Magn. Magn. Mater. 35, 219-220 (1983).

14. Jang, K.-l. et al. Rugged and breathable forms of stretchable electronics with adherent composite substrates for transcutaneous monitoring. Nat. Commun. 5, 4779 (2014).
15. Pan, L. et al. An ultra-sensitive resistive pressure sensor based on hollow-sphere microstructure induced elasticity in conducting polymer film. Nat. Commun. 5, 3002 (2014).

16. Tien, N. T. et al. A flexible bimodal sensor array for simultaneous sensing of pressure and temperature. Adv. Mater. 26, 796-804 (2014).

17. Choong, C.-L. et al. Highly stretchable resistive pressure sensors using a conductive elastomeric composite on a micropyramid array. Adv. Mater. 26, 3451-3458 (2014).

18. Kim, J. et al. Stretchable silicon nanoribbon electronics for skin prosthesis. Nat. Commun. 5, 5747 (2014).

19. Matsuhisa, N. et al. Printable elastic conductors by in situ formation of silver nanoparticles from silver flakes. Nat. Mater. 16, 834 (2017).

20. Boutry, C. M. et al. A sensitive and biodegradable pressure sensor array for cardiovascular monitoring. Adv. Mater. 27, 6954-6961 (2015).

21. Jung, M. et al. Vertically stacked nanocellulose tactile sensor. Nanoscale 9, 17212-17219 (2017).

22. Kim, K. et al. Low-voltage, high-sensitivity and high-reliability bimodal sensor array with fully inkjet-printed flexible conducting electrode for low power consumption electronic skin. Nano Energy 41, 301-307 (2017).

23. Jung, M. et al. Paper-based bimodal sensor for electronic skin applications. ACS Appl. Mater. Interfaces 9, 26974-26982 (2017).

24. Meng, L.-J. \& dos Santos, M. P. Investigations of titanium oxide films deposited by dc reactive magnetron sputtering in different sputtering pressures. Thin Solid Films 226, 22-29 (1993).

25. Ketov, S. V. et al. Formation of nanostructured metallic glass thin films upon sputtering. Heliyon 3, e00228 (2017).

26. Löbl, P., Huppertz, M. \& Mergel, D. Nucleation and growth in TiO2 films prepared by sputtering and evaporation. Thin Solid films 251, 72-79 (1994).

27. Park, J., Kim, M., Lee, Y., Lee, H. S. \& Ko, H. Fingertip skin-inspired microstructured ferroelectric skins discriminate static/dynamic pressure and temperature stimuli. Sci. Adv. 1, e1500661 (2015).

28. Fan, J. A. et al. Fractal design concepts for stretchable electronics. Nat. Commun. 5, 3266 (2014).

29. Zandman, F. \& Szwarc, J. Non-linearity of resistance/temperature characteristic: its influence on performance of precision resistors. Vishay Precision Group, Tech. Rep 108 (2013).

30. Jeon, S. et al. Gated three-terminal device architecture to eliminate persistent photoconductivity in oxide semiconductor photosensor arrays. Nat. Mater. 11, 301-305 (2012).

31. Jeon, S. et al. Origin of high photoconductive gain in fully transparent heterojunction nanocrystalline oxide image sensors and interconnects. Adv. Mater. 26, 7102-7109 (2014).

32. Park, K. et al. Stretchable, transparent zinc oxide thin film transistors. Adv. Funct. Mater. 20, 3577-3582 (2010).

33. Kaltenbrunner, M. et al. An ultra-lightweight design for imperceptible plastic electronics. Nature 499, 458 (2013).

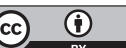

Open Access This article is licensed under a Creative Commons Attribution 4.0 International License, which permits use, sharing, adaptation, distribution and reproduction in any medium or format, as long as you give appropriate credit to the original author(s) and the source, provide a link to the Creative Commons license, and indicate if changes were made. The images or other third party material in this article are included in the article's Creative Commons license, unless indicated otherwise in a credit line to the material. If material is not included in the article's Creative Commons license and your intended use is not permitted by statutory regulation or exceeds the permitted use, you will need to obtain permission directly from the copyright holder. To view a copy of this license, visit http://creativecommons. org/licenses/by/4.0/.

(c) The Author(s) 2019 